\title{
What is the Effective Geometrical Collection Efficiency of Your XEDS Detector? A Routine Procedure Applied in a SEM Laboratory.
}

\author{
Nanthawan Avishai ${ }^{1, *}$, Amir Avishai ${ }^{1}$ and Vasile-Dan Hodoroaba ${ }^{2}$ \\ 1. Swagelok Center for Surface Analysis of Materials, Department of Materials Science and Engineering, \\ Cleveland, OH, USA. \\ 2. Federal Institute for Materials Research and Testing (BAM), Division 6.1 Surface Analysis and \\ Interfacial Chemistry, Berlin, Germany.
}

At the Microscopy and Microanalysis 2015 meeting, two contributions dealt in detail with the assessment of the geometrical collection efficiency (GCE) of energy dispersive X-ray spectrometer (EDS) [1, 2]. While the Schamber's approach [1] focussed on the practical determination of the GCE, expressed as "kilocounts/second/nanoamp", by measuring $\mathrm{Cu} \mathrm{K}$ series X-rays, the second approach proposed by Procop et al. [2] goes further and calculates the GCE (or the corresponding detector active area) from the measured count rates compared to theoretical values. The key parameter necessary for the theoretical evaluation of the GCE is the X-ray generation yield [in photons $/ \mathrm{nC} / \mathrm{msr}$ ] for $\mathrm{Cu} \mathrm{K} \alpha$ radiation. From the few reports available in the literature, e.g. [3], an approximate value of 600 photons $/ \mathrm{nC} / \mathrm{msr}$ for $\mathrm{Cu} \mathrm{K \alpha}$ radiation is used for the calculation of the $20 \mathrm{kV}$ X-ray spectra, so that by comparison with measured spectra, the true solid angle can be extracted. The associated measurement uncertainty is below $10 \%$. The absorption correction and spectrometer efficiency of $\mathrm{Cu} \mathrm{K \alpha}$ radiation are not critical parameters. However, care must be taken in the accurate measurement of the beam current; the use of a calibrated pico-ammeter and a Faraday cup constitute the preferred solution. Hence, the determination of the true solid angle, or, equivalently, the effective illuminated area, of an EDS detector may be applied as a metrological tool for the routine evaluation of EDS performance by users in any service laboratory. This could complement the periodic check of EDS performance in compliance with the well-established international standard ISO 15632:2012 [4].

The practical calculation of the nominal $\mathrm{Cu} \mathrm{K \alpha}$ count rate (normalized to current) as measured by e.g. a $10 \mathrm{~mm}^{2} \mathrm{EDS}$ at a $40 \mathrm{~mm}$ distance from specimen is calculated as follows: 600 photons $/ \mathrm{nC} / \mathrm{msr}$ (X-ray yield at $20 \mathrm{kV}) \times 0.97$ (absorption correction factor) x 0.78 counts/photons (EDS efficiency) $\times\left(10 / 40^{2} \mathrm{x}\right.$ $1000) \mathrm{msr}($ solid angle $)=2.8 \mathrm{kilocounts} / \mathrm{s} / \mathrm{nA}$. Relating this value to that resulting from the measurement at $40 \mathrm{~mm}$ distance leads to the exact fraction of detector area (or solid angle) effectively illuminated under the given conditions.

In this contribution, two large-area EDS detectors were tested according to the procedure proposed in [2]. In a first step, the optimal working distance (WD) in the two different SEM chambers was determined by moving the sample stage in the $\mathrm{Z}$ direction and monitoring the count rates at a magnification of 10,000 and a field of view of $25.6 \mu \mathrm{m}$ (Figure $1 \mathrm{~A}$ ). The WD at which the highest intensity was measured was selected as the optimal position, corresponding to the crossover between the EDS detector optical axis and electron beam optical axis. Next the $\mathrm{Cu} \mathrm{K \alpha}$ peak was measured at different relative EDS positions while it was partially removed from the fully inserted position. The spectrum at each location was collected for $10 \mathrm{sec}$ (live time) using the highest pulse rate and intermediate current ( $2.3 \pm 0.1 \mathrm{nA})$ to minimize pile up effects (13\% dead time). The 'inverse squared normalized intensities vs. relative EDS position' used to extract the true detector - specimen distance, as described in [2], shows a non-linear relationship (Figure $1 \mathrm{~B})$ even at the minimal relative positions, which indicates shadowing due to obstruction or use of an 
unsuitable and/or off-centered collimator. The normalized count rates measured as a function of the EDS distances, results in a too low GCE (too low true solid angles) for both tested detectors. EDS \#1 and EDS $\# 2$ : effectively use only a fraction of the nominal detector area $17 \%$ and $12 \%$, respectively. The search for sources of losses of signal due to possible shadowing effects is in progress.

In conclusion, the procedure proposed by Procop et al. [2], to determine the true solid angle of an EDS detector on a metrological basis is a valuable practical approach to evaluate signal loss due to potential EDS misalignment in the SEM chamber, possibly occurring after the installation or faulty collimation of the X-rays at the entrance of the EDS detector.

\section{References:}

[1] F Schamber, Microsc. Microanal. 21 (Suppl 3) (2015), p. 1479.

[2] M Procop et al., Microsc. Microanal. 21 (Suppl 3) (2015), p. 1481.

[3] M Procop, Microsc. Microanal. 10 (2004), p. 481.

[4] ISO 15632:2012, "Microbeam Analysis - Selected instrumental performance parameters for the specification and checking of energy-dispersive X-ray spectrometers for use in electron probe microanalysis", ISO: Geneva.

[5] We would like to acknowledge the help of Dr. M Procop (Berlin, Germany) in application of the measurement procedure and drafting the abstract and Prof. Arthur Heuer (Case Western Reserve University) for his critical reviewing and editing of the manuscript.
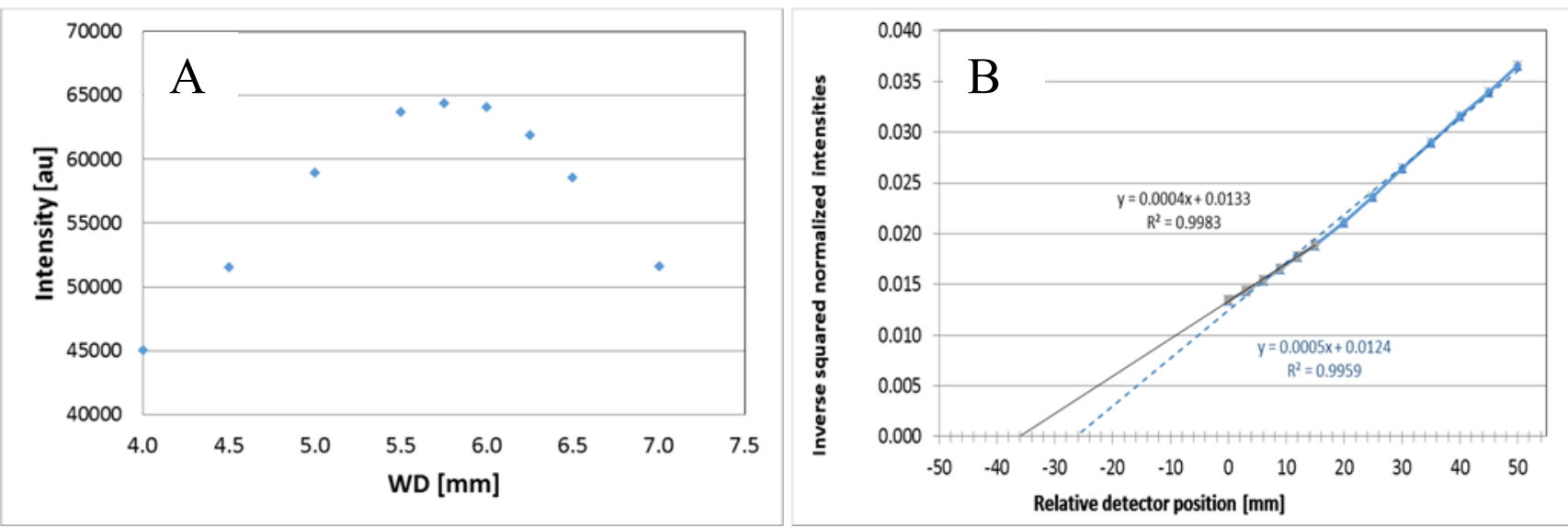

Figure 1. A) Measured intensity of $\mathrm{Cu} \mathrm{K} \alpha$ as a function of WD (EDS \#1) and (B) determination of the absolute detector specimen distance (EDS \#1). Note the various possible cut-offs of the abscissa due to the non-linearity of the curve. The grey trend line represents a fit of the closest six points while the blue dashed trend line represents the linear fit of the full data set. 